\title{
The Design and Evaluating Method for The Scanning Strategy in Nano- second Laser Milling of Axisymmetric 3D Structure Micro Cone Consid- ering Its Symmetric Errors
}

\author{
Xiong-dun Xie, Yang-zhi Chen and Yan-jie Shao \\ School of Mechanical and Automotive Engineering, South China University of Technology, 381 \\ Wushan Rd, Tianhe, Guangzhou 510640, China \\ E-mail: meyzchen@scut.edu.cn
}

\begin{abstract}
Nanosecond laser milling has significant thermal effects, as a result, the shape accuracy of the manufactured microstructures is poor. At present, grooves, channels and slots are the most common objects in the studies on the effects of process parameters, such as laser power, laser frequency, scanning speed, on laser milling processing effects. However, the influences of scanning strategy on shape errors of micro 3D structures have rarely been researched. This paper focuses on the scanning strategy in nanosecond laser milling of axisymmetric 3D structures micro cones, on which symmetric errors depend. Firstly, the material removal calculation of nanosecond laser ablation is conducted and a design method on the scanning strategy of laser milling manufacturing of micro cones is proposed; Then, the evaluating method of scanning strategy is proposed where symmetry coefficient is used to quantify the spatial symmetry degree of scanning strategies; Finally, experiments are carried out to prove the validity of the proposed evaluation method of scanning strategy. The design and evaluating method of the scanning strategy in nanosecond laser milling of axisymmetric 3D structure are reasonable and are capable of being applied in the future.
\end{abstract}

DOI: $10.2961 /$ jlmn.2020.01.2006

Keywords: micro manufacture, nanosecond laser milling, scanning strategy, micro cone, shape accuracy

\section{Introduction}

Laser milling is a micro manufacturing technology that can be applied to machining of 3D structures[1][2]. The advantages of laser milling include non-contact material removal, adaptability to wide range of materials, ability to realize small structure sizes, convenience of integrating with $\mathrm{CNC}$ system, while the disadvantages include low energy utilization and processing efficiency, the existence of heat affected zone (HAZ) and recast layer, and complicated process parameters[2]. Femtosecond and picosecond laser milling have very high machining accuracy and little thermal effect, but low material removal rate (MRR) and expensive equipment[3][4], so they are suitable for high value-added products and micro structures with overall sizes smaller than $100 \mu \mathrm{m}$. Only when the processing efficiency, precision and cost of a micro manufacturing technology has a comprehensive advantage can it has practical application value. Nanosecond laser milling is suitable for machining micro structures with overall sizes larger than $100 \mu \mathrm{m}$, for example, micro cones[5][6], micro grooves[7] and micro parts[2][8], because it has a comprehensive advantage of material removal efficiency and processing accuracy at this scale, and its equipment is not expensive.

The material removal in nanosecond laser ablation is based on thermal effect, so it has a poor machining accuracy and can only be used as a rough machining process[9]. In order to achieve the ideal processing effect, first, it is necessary to select optimal process parameters to meet a certain standard of shape accuracy, and then secondary finishing process and post-treatment process can be carried out. Our work focuses on the first step.

Laser milling has many process parameters, including but not limited to laser power, laser frequency, scanning speed, spacing of scanning path and scanning strategy. Existing researches focused on the influences of process parameters on laser milling processing effect, as well as the improvement of processing effect through the collocation and optimization of process parameters. The researchers concerned with MRR, surface roughness, groove width and aspect ratio, and their optimization goals include increasing the MRR, reducing the surface roughness, the thickness of HAZ and recast layer. The objects of the studies are mostly simple structures such as channels[10] and grooves[7][11][12]. For example, Feng. W. et al explored the influence of the combination of laser power and scanning speed on the roughness of the bottom of the ablation groove, and selected a better parameter interval[11]; Sivarao et al , Mandal K.K. et al used respond surface method (RSM) or Tachi method to select better parameters for processing grooves with a smooth bottom[13][14].

Among the process parameters, scanning strategy is non-negligible especially in manufacturing of 3D structures. Scanning strategy refers to the setting rules of scanning trajectories, including scanning directions, number of scans, scanning sequence and the scanning rules in different regions. At present, there are a few studies on the influences of scanning strategy on laser milling processing effect and the design of scanning strategies. For example, Naveed 
Ahmed et al considered scanning mode of laser milling of difficult-to-cut Alloys[15], Stefan Janssen et al[16] proposed an improved scanning strategy to reduce thermally induced defects in drilling of carbon fiber textiles. So far, to our very knowledge, no study focuses on the evaluating method for scanning strategy in laser milling process in consideration of shape errors of 3D microstructures and its application to manufacturing of axisymmetric micro structures.

This paper proposed an evaluating method for scanning strategy in nanosecond laser milling of axisymmetric 3D structure. Micro cone with size range of $600-900 \mu \mathrm{m}$ is used as a validation example of the proposed method. First, the material removal calculation of nanosecond laser ablation is derived and the technology of nanosecond laser milling of microcone is introduced. Then, in order to quantitatively evaluate the spatial symmetry degree of scanning strategy for machining of symmetrical 3D structures, the symmetry coefficient $S Y M$ of laser milling scanning strategy is proposed. Finally, it is proved by experiments that the symmetry of the micro cones is positively correlated with the symmetry coefficient SYM of the scanning strategy. The evaluation method of scanning strategy is reasonable to the formulation of scanning strategy of laser milling of micro cones or other symmetric micro 3D structures and to reduce shape errors.

\section{Nanosecond laser milling process for micro cones 2.1 Material removal of nanosecond laser ablation}

In the case of nanosecond laser ablation of metal, the material is removed by thermal effect, and the main mechanisms are normal evaporation and phase explosion[17][18]. The energy density distribution of a single laser pulse on the surface of the target is expressed by Eq.(1)[19].

$I(t, r)=I_{p k}\left(\frac{t}{t_{p k}}\right)^{n} \exp \left(n\left(1-\frac{t}{t_{p k}}\right)\right) \exp \left(-\frac{r^{2}}{2 r_{\max }^{2}}\right), 0 \leq t \leq t_{\max }$

Where, $t, t_{p k}$ and $t_{\text {max }}$ denote some time after a pulse starts, the time when the pulse reaches its peak and the width of the pulse respectively; $r$ and $r_{\max }$ denote the distance from the center of the laser spot and the spot radius respectively; $I_{p k}$ denotes the peak power of the pulse; and $n$ is a laser parameter that decides the shape of the pulse.

The material removal process of nanosecond laser ablation can be approximately described by the Stefan problem with moving boundaries. Ref [20] provided the analytical solution of the Stefan problem: With the given conditions of target material's thermal parameters and the power input described in Eq.(1), $v(t, r)$, the moving velocity of the evaporation boundary can be calculated. And then, by integrating $v(t, r)$ over the time of a laser pulse, the position of evaporation interface at the end of laser radiation on the target is obtained: $z\left(t_{\max }, r\right)=\int_{0}^{t_{\max }} v(t, r) d t$. According to the ablation morphology results of a large number of experiments, the evaporation interface $z\left(t_{\max }, r\right)$ can be well fitted by Gaussian function $z_{g}(r)$, as shown in Eq.(2). $a$ and $b$ are the parameters.

$$
z=z_{g}(r)=a \exp \left(\frac{-r^{2}}{2 b^{2}}\right)
$$

To calculate the crater's volume, the formula of volume of the body of revolution is used, as shown in Eq.(3)

$$
V={ }_{x_{\min }}^{x_{\max }} p(f(x))^{2} d x
$$

The axis of rotation is $\mathrm{x}$ axis in Eq.(3), while the axis of symmetry of Eq.(2) is $\mathrm{z}$ axis. So the independent and dependent variables of Eq.(2) need to be exchanged before Eq.(3) can be applied.

As shown in Eq.(4), $V_{0}$ is the volume of single crater. $r_{b}$ denotes the maximum radius where the target can reach the boiling temperature in a laser pulse, and the material removal only occurs at $r \in\left(0, r_{b}\right)$.

$$
V_{0}=\int_{z_{g}\left(r_{b}\right)}^{z_{g}(0)} \pi\left(\sqrt{-2 b^{2} \ln \left(\frac{z_{g}(r)}{a}\right)}\right)^{2} d z
$$

\subsection{Manufacture technique of micro cones}

In nanosecond laser milling, the laser spot is controlled to move relative to the blank by using galvanometer or motion platform or a combination of the two ways, and target material at different position of the blank can be removed, which is the superposition of single laser pulse ablation. The laser milling principle of micro cone is shown in Fig. 1(a). The cone is sliced and processed by step fitting. As shown in Fig. 1(b), a series of concentric rings can be obtained by projecting the fitted steps onto the horizontal plane, and these rings are the scanning regions. In laser milling process, material is removed layer by layer in these concentric ring areas according to a certain scanning strategy and finally a cone is formed. Specifically, at each layer, the scan starts at the outside ring and ends at the inside ring. The number of scans of the ring-shape regions from outside to inside is reduced, while the central circle area is not scanned, so the material removal is gradually reduced from the outside to the inside to form the target cone.
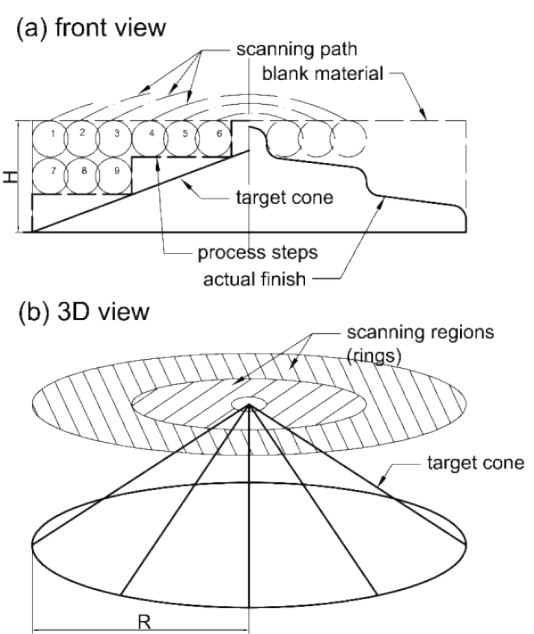

Fig. 1 Laser milling process of micro cone. 
According to the material removal calculation of Eq.(4), using the above method, the cone angle of the manufactured cone is calculated by Eq.(5):

$$
\theta=2 \arctan \left(\frac{R}{H}\right)=2 \arctan \left(\frac{R h v}{n_{1} n_{2} q V_{0}}\right)
$$

Where, $R$ denotes the radius of bottom circle and $H$ denotes the removal depth of the outer ring as marked on Fig. $1 ; n_{1}$ denotes the number of layers; $n_{2}$ denotes the number of scans per layer; $v$ denotes the scanning speed; $h$ denotes the spacing of scanning path and $q$ denotes the laser frequency. A micro cone is obtained by using the superposition of the ideal material removal in Eq.(4), without considering the scanning speed and direction, the overlap of ablation that will cause uneven material removal and material sputtering, and without considering the heat accumulation that will also affect material properties, in turn, influence the MRR, and the effect of thermal deformation. Therefore, Eq.(5) can only make a rough estimation on the cone shape.

\subsection{Spatial symmetry of scanning strategy}

In the laser milling of axisymmetric 3D structures, such as cones, circular platforms and hemispheres, the material to be removed are also axisymmetric. Therefore, the easiest way to manufacture axisymmetric $3 \mathrm{D}$ structures is to scan several axisymmetric regions. In this article, all the scanning regions in the scanning strategies refer to axisymmetric regions.

Micro cone is the machining example in this paper. The shape errors of a cone include cone angle error, diameter error and symmetric error. Some technology factors causing the above errors cannot be eliminated: First, the equipment errors, such as the fluctuation of the laser output, the fluctuation of the scanning speed of the galvanometer and the positioning error of the motion platform; Second, asymmetric factors of temporal distribution of energy that comes from the scanning principle: the material at different positions is ablated following a sequential order, resulting in asymmetric shape of targets in the milling process, so there are differences in the thermal conduction and material removal rate during processing.

Although the equipment errors and the asymmetry of temporal distribution of energy cannot be eliminated, the asymmetry of spatial distribution of energy can be reduced, among which the most important factor is the scanning strategy. By designing scanning strategies with strong spatial symmetry, symmetric error of symmetrical 3D structures can be reduced. In order to quantify the degree of spatial symmetry of a scanning strategy for axisymmetric $3 \mathrm{D}$ structures, an evaluating method is proposed. The derivation is as follows:

A laser milling process of axisymmetric 3D structures is consisted of several scans in one region or in several regions. In each scan, the laser spot moves along the scanning path of a certain direction according to the set process parameters. An asymmetric effect on the processed area is produced, which is defined as scanning orientation effect. As shown in Eq.(6), $\vec{D}$ is defined as the vector of scanning orientation effect, the orientation effect vector for short. $E$ denotes the energy density of the scanning process,
$S$ denotes the processed area and $\overrightarrow{p a t h}$ is a unit vector representing the direction of the scanning path.

$$
\vec{D}=E S \overrightarrow{\text { path }}
$$

In a laser milling process, there are several scanning orientation effects, corresponding to several $\vec{D}$, as shown in Eq.(7), where, $n$ is the number of orientation effect vector.

$$
\overrightarrow{D_{i}}=E_{i} S_{i} \overrightarrow{p a t h}_{i},(i=1,2, \ldots, n)
$$

First, the simplest case is considered: $E_{i}=$ const , $S_{i}=$ const , which means $\left|\vec{D}_{i}\right|=$ const . An example is demonstrated in Fig. 2(a).In this case, $S Y M$, the symmetry coefficient, is the ratio of the sum of the areas of triangles surrounded by adjacent $P_{i},(i=1,2,3,4)$ and $O$ (the shadow area) to the area of the circle with the radius of $|\vec{D}|$. The calculation is shown in Eq.(8). $S Y M \in[0,1]$.

$$
S Y M=\left\{\begin{array}{c}
0 \quad, n=1 \\
\frac{\sum_{i=1}^{n-1} S_{\triangle O P_{i} P_{i+1}}+S_{\triangle O P_{n} P_{1}}}{\pi\left(\left|\vec{D}_{\text {const }}\right|\right)^{2}}, n>1 \\
\left(E_{i}=\text { const }, S_{i}=\text { const }\right)
\end{array}\right.
$$

In a common case: $E \neq$ const and $S \neq$ const, which means $\left|\vec{D}_{i}\right| \neq$ const, $S Y M$ is calculated by Eq.(9).

$$
S Y M=\left\{\begin{array}{ccc}
0 & , n=1 \\
\frac{\sum_{i=1}^{n-1} S_{\triangle O P_{i x} P_{i+1 x}}+S_{\triangle O P_{n x} P_{1 x}}}{\pi \max \left(\left|\vec{D}_{i}\right|\right)^{2}} \times \frac{\sum_{i=1}^{n} \sum_{j=1}^{n, j \neq i} S_{\triangle O P_{i} P_{j}}}{\sum_{i=1}^{n} \sum_{j=1}^{n, j \neq i} S_{\triangle O P_{i} P_{j x}}}, n>1
\end{array}\right.
$$

An example is demonstrated to show the principle of Eq.(9). As can be seen in Fig. 2(b), $O$ is the starting point of $\overrightarrow{D_{i}},(i=1,2,3,4)$ and $P_{i},(i=1,2,3,4)$ are their ending points. A circle is formed with the radius of $\max \left(\left|\overrightarrow{D_{i}}\right|\right)$ which is $\left|\overrightarrow{D_{1}}\right|$ in the example, and $\overrightarrow{D_{i}},(i=2,3,4)$ are extended until they intersect with the circle at $P_{i x},(i=2,3,4)$, while $P_{1}$ and $P_{1 x}$ are the same point. Assuming that $\left|\overrightarrow{D_{i}},(i=2,3,4)\right|=\left|\overrightarrow{D_{1}}\right|$, then according to Eq.(8) , $S Y M=\left(\sum_{i=1}^{n-1} S_{\triangle O P_{i x} P_{i+1 x}}+S_{\triangle O P_{n x} P_{1 x}}\right) / \pi \max \left(\left|\vec{D}_{i}\right|^{2}\right)$. In the common case, $\left|\vec{D}_{i}\right| \neq$ const , a reduction coefficient $\sum_{i=1}^{n} \sum_{j=1}^{n, j \neq i} S_{\triangle O P_{i} P_{j}} / \sum_{i=1}^{n} \sum_{j=1}^{n, j \neq i} S_{\triangle O P_{i} P_{j x}}$ need to be multiplied. 
(a)

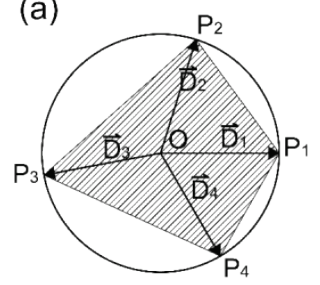

(b)

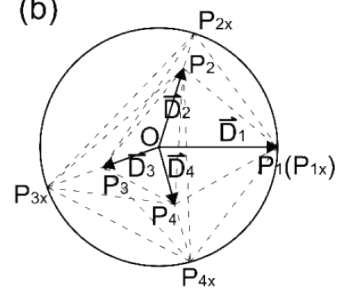

(a) multiple orientation effect vectors with a same module (b) multiple orientation effect vectors with different modules

Fig. 2 Demonstration of $S Y M$.

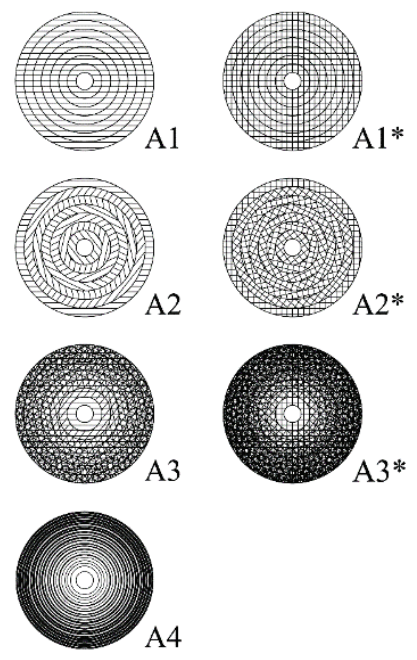

Fig. 3 Seven scanning strategies.

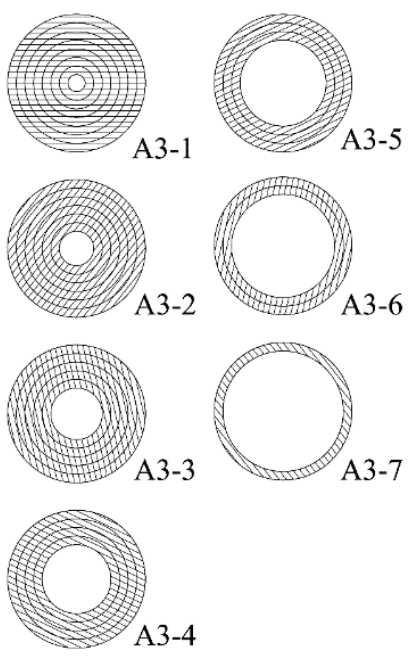

Fig. 4 Decomposition of A3.

In this paper, the design of the scanning strategy follows a setting that is easy to be realized by the laser equipment, that is, the spacing of scanning paths in a scanning strategy is uniform, and the process parameters including laser power, laser frequency, scanning speed, spacing of scanning path, remain unchanged along all scanning paths,. That means $E=$ const along the whole machining process.

Fig. 3 shows the scanning paths of seven scanning strategies for machining a cone. The total lengths of their scanning paths are equal, which means, the processing efficiencies are equal. $k$ is the number of rings to be scanned, in the examples of Fig. 3, $k=7$. The scanning rules of seven scanning strategies are presented as follows:

In A1, the internal scanning path of each ring is horizontal; In A2, the scanning path inside each ring has a different angle, the ith ring counting from outside has the angle of $360^{\circ}(i-1) / k,(i \in[1, k])$; In $\mathrm{A} 3$, the scanning path of each layer has a different angle, the $j$ th layer has the angle of $360^{\circ}(j-1) / k,(j \in[1, k])$; In A4, all the scanning paths are circles.

To facilitate the understanding of the "layers" of the scanning strategy, Fig. 4 shows the decomposition of A3 of Fig. 3: A3 is superimposed of 7 layers of A3-1 A3-7, and the processing sequence is $\mathrm{A} 3-1 \sim \mathrm{A} 3-7$. A1 and $\mathrm{A} 2$ are also superimposed of 7 layers, it's just that the scanning paths within each ring are the same, so the patterns of the scanning paths overlap.

The number of scans within the rings increases from inside to outside, the $i t h$ ring has the number of scans of $2 k-2(i-1) . \mathrm{A} 1 *, \mathrm{~A} 2 *$ and $\mathrm{A} 3 *$ are the corresponding modified schemes of A1, A2 and A3, of whom the scanning direction of the second scan of each layer is set as the vertical direction of the original ones.

According to Eq.(9), substituting the parameters in table 1, SYM of the seven scanning strategies are obtained as follows:

$$
\begin{aligned}
& S Y M(\mathrm{~A} 1)=0 ; \operatorname{SYM}(\mathrm{A} 2)=0.5248 ; \operatorname{SYM}(\mathrm{A} 3)=0.6406 ; \\
& \operatorname{SYM}\left(\mathrm{A} 1^{*}\right)=0.1592 ; \operatorname{SYM}(\mathrm{A} 2 *)=0.5503 ; \\
& S Y M\left(\mathrm{~A} 3^{*}\right)=0.6679 ; \operatorname{SYM}(\mathrm{A} 4)=1 .
\end{aligned}
$$

According to the above results, the symmetric errors of micro cones processed by $\mathrm{A} 1$ and $\mathrm{A} 1 *$ may be larger, while the symmetric errors may be smaller when processed by $\mathrm{A} 2, \mathrm{~A} 3, \mathrm{~A} 2 *$ and $\mathrm{A} 3 *$, and micro cones processed by A 4 may have the smallest symmetric errors. In addition, by longitudinal comparison, the symmetric errors of micro cones processed by scanning strategies with * may be smaller than that without *, that is, $\mathrm{A} 1 *, \mathrm{~A} 2 *$ and $\mathrm{A} 3 *$ are superior to A1, A2 and A3 respectively.

\section{Experiments}

\subsection{Experiment setups and measuring method}

The model of the nanosecond laser milling equipment is HM20 laser marking machine (HAN'S LASER), equipped with MOPA laser with wavelength of $1.06 \mu \mathrm{m}$, power of $20 \mathrm{~W}$, laser repetition frequency of $1-1000 \mathrm{kHz}$, and photoelectric galvanometer with repetition accuracy of $3 \mu \mathrm{m}$. The transmitted beam is focused on the target surface by using of a set of lenses in brand of Carman Hass SL1064-110-160.The measuring device is VHX-2000 ultrafine field microscope with magnification of $100 \times-1000 \times$. The surface topography of 3D structures can be obtained, and the $3 \mathrm{D}$ coordinates of the measured work piece can be saved through the built-in software. The target material is 6061 aluminum alloy (AlMg1SiCu ISO 209.11989). 
Measuring methods of micro cone shape accuracy are as follows: after obtaining the 3D morphology of a micro cone, the morphologies of 12 base lines with equal angle distribution on a micro cone surface are extracted, then the shape accuracies including average cone angle, symmetric error and diameter error of micro cones are calculated. Average cone angle is calculated by the slope of the fitting line of 12 base lines morphologies; Symmetric error is equal to the average distance between the 12 base lines morphologies and the average base line. The smaller the value, the better the cone symmetry; Diameter error is equal to the maximum deviation of 12 base lines morphologies from a straight line.

The seven scanning strategies in section 2 are applied to manufacture micro cones to confirm whether the cone shape accuracy is in line with the analytical result in section 2. The process parameters are shown in Table 1. Group 1 has a smaller spacing of the scanning path, which means the energy density of processing in group 1 is larger than that of group 2. According to the measuring methods, the symmetric errors are compared, and the cone angle and diameter error are also concerned.

Table 1 Process parameters

\begin{tabular}{c|c|c}
\hline group & 1 & 2 \\
\hline spacing of path & $3 \mu \mathrm{m}$ & $5 \mu \mathrm{m}$ \\
\hline power & $12 \mathrm{~W}$ \\
\hline scanning speed & $1000 \mathrm{~mm} / \mathrm{s}$ \\
repetition frequency & $20 \mathrm{kHz}$ \\
number of layers & 12 \\
\hline cone diameter $(\mu \mathrm{m})$ & D600, D700, D800, D900 \\
\hline
\end{tabular}

\subsection{Results}

The data of machining results are shown in Fig. 5 and Fig. 6. The topographies of the micro cones are shown in Fig. 7, which is an example of D800 in group 2, while other groups have the similar characteristics. Fig. 8 is the corresponding figure of Fig. 7. It shows the cross-sections' shape of micro cones. In the figures, the color represents the height of the 3D structures, with red being the highest and blue the lowest. In the experiments, the processing time of the seven scanning strategies is equal.

The results of average cone angles of micro cones for group 1 and 2 are shown from Fig. 5(a) and (b) respectively. The average cone angles of $\mathrm{A} 1, \mathrm{~A} 2$ and $\mathrm{A} 3$ are larger than that of $\mathrm{A} 1 *, \mathrm{~A} 2 *$ and $\mathrm{A} 3 *$, and the average cone angles of A4 are the smallest except for D600 in group 2. All eight curves reach their peak values at $\mathrm{A} 2$, which means the scanning strategy has a certain influence on the average cone angle.

The results of symmetric errors shown in Fig. 6 are the focus of this paper because they are directly related to $S Y M$ in section 2. According to the data in Fig. 6 (a) and (b), the symmetric errors for $\mathrm{A} 1$ and $\mathrm{A} 1 *$ are the largest, while those for $\mathrm{A} 2, \mathrm{~A} 3, \mathrm{~A} 2 *$ and $\mathrm{A} 3 *$ are smaller, and those for A4 are the smallest.

The diameter errors of the micro cones are also calculated. The results are within the range of $30-120 \mu \mathrm{m}$, which are very large. Preliminary analysis proved that diameter error on micro cone varies with cone size, laser power density and scanning strategy.

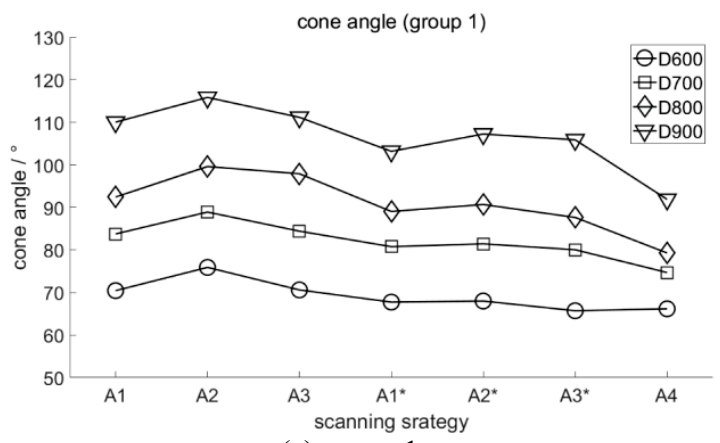

(a) group 1

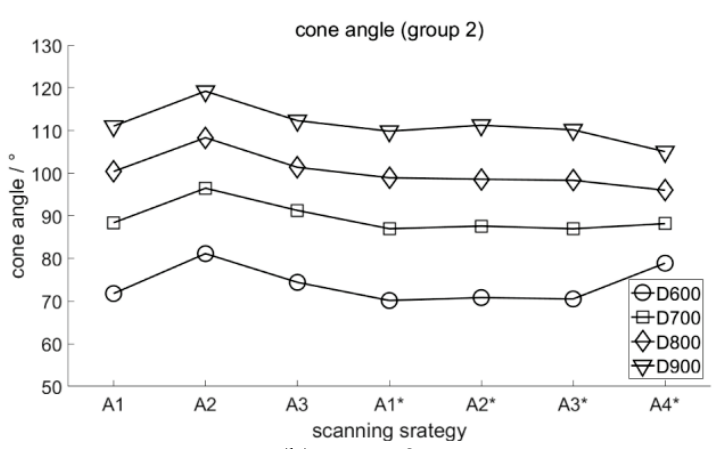

(b) group 2

Fig. 5 Cone angle.

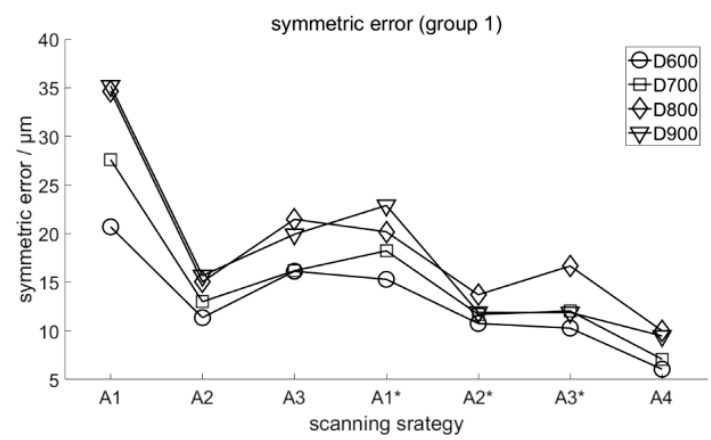

(a) group 1

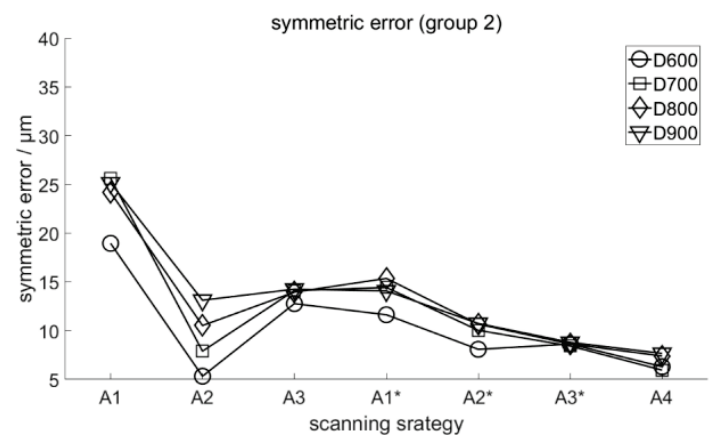

(b) group 2

Fig. 6 Symmetric error. 


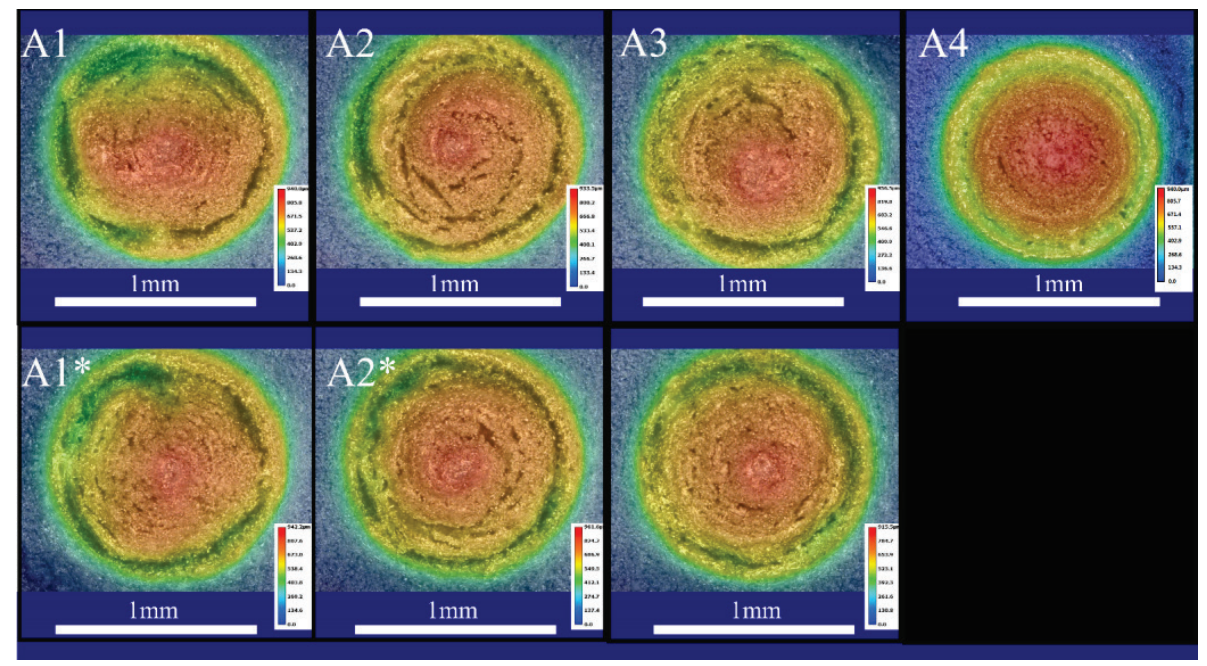

Fig. 7 Topographies of D800 in group 2.

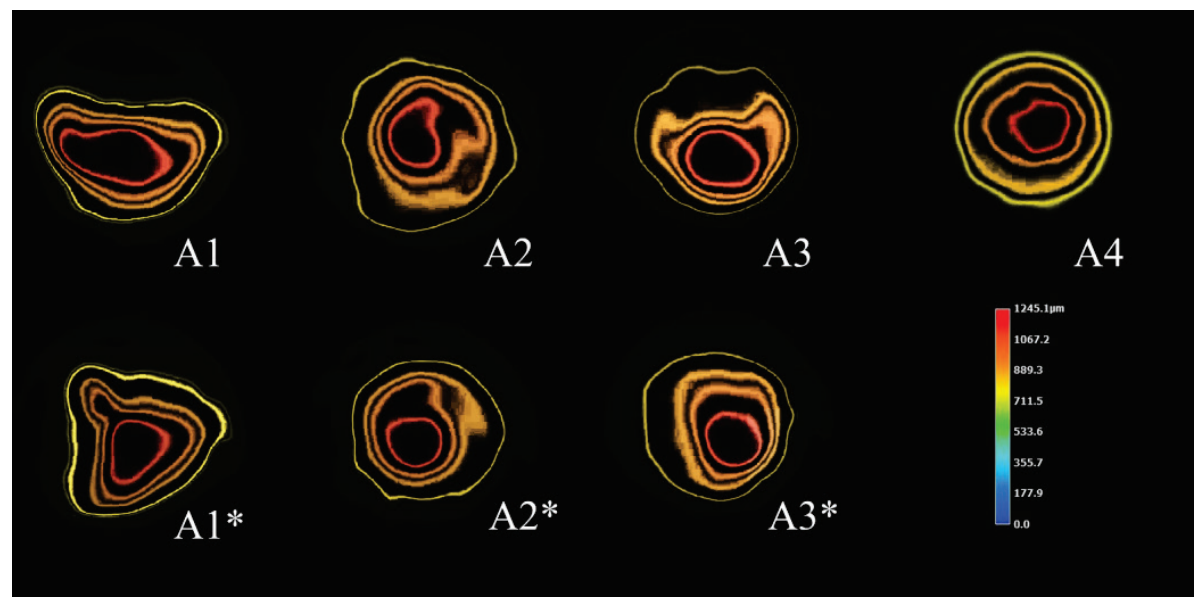

Fig. 8 Cross-sections shape of micro cones of D800 in group 2.

\subsection{Analysis and discussion}

The results of the experiments are analyzed and discussed in this section.

By comparing the curves in Fig. 5(a) and (b) separately, it can be seen that when other parameters stay the same, the average cone angle increases with the radius of the bottom surface of the cone; By comparing the corresponding curves in Fig. 5(a) and (b), the average cone angle increases with the increase of the spacing of the scanning path. The regularities of the cone angles of different diameters and spacing of scanning path shown in Fig. 5 conform to Eq.(5). Although Eq.(5) is just an estimation, it reveals the relationship between process parameters and cone angle.

As shown in Fig. 6(a) and (b), the symmetric error is strongly affected by laser power density and scanning strategy. At every corresponding point, the symmetric error of group 2 is lower than that of the group 1 which mean the symmetric error increases as the increase of laser power density; In each group, A1 and A1* have the largest symmetric errors followed by A3, A2, A2* and A3*, and A4 has the lower value. It can be shown by Fig. 7 that among the processing results, $\mathrm{A} 1$ and $\mathrm{A} 1 *$ have the poorest symmetry and A4 is the most homogeneous, the most symmetrical, and with the least flawed.
Fig. 8 shows the cross-sections' shape of micro cones of D800 in group 2.A1 is processed by horizontal scanning. Therefore, the overall shape of the cone is flat, so that its symmetry is very poor. The cone for A $1 *$ is scanned horizontally and vertically, and the overall shape of the cone has two corresponding protrusions. The contour line is somewhat close to the triangle, and the symmetry is also very poor. The cones for A2, A3, A2*, A3* adopted multidirection scanning, so their symmetry accuracies are greatly improved, but the contour line was still eccentric to a certain extent. Among the seven results, A4 should be completely symmetrical theoretically, because it is processed by circle scanning which is perfectly symmetrical. However, because of the asymmetry of temporal distribution of energy and the fluctuation of the laser output the contour lines of A4 are not totally circular.

Fig. 9 shows data of the SYM of scanning strategies and the mean symmetric errors of the processed micro cones. The dotted and dash-dotted lines in the figure are the trend lines of two groups. With the increasing of $S Y M$, the processed micro cone has a smaller symmetric error. $S Y M$ of the scanning strategy has a significant negative correlation with the symmetric error of the processed cone, 
so the evaluating method on basis of SYM can reasonably predict the symmetry of micro cone and it may be extended to evaluating of scanning strategies for other symmetric 3D structures.



Fig. 9 Symmetric error $-S Y M$.

The cause of diameter errors is thermal effect in nanosecond laser milling, specifically, the surface thermal deformation and sputtering of material. At present, the diameter errors are very large, and even the best result is over $30 \mu \mathrm{m}$. Diameter error is influenced by multiple factors including cone size, laser power density and scanning strategy and its mechanism is still unclear. To further improve the diameter error of the micro cones, the mechanism of diameter error and secondary finishing process need future studies.

\section{Conclusions}

Due to thermal effect, the shape accuracy of $3 \mathrm{D}$ micro structures processed by nanosecond laser milling is poor. The scanning strategy has a significant effect on the symmetric error of the micro axisymmetric $3 \mathrm{D}$ structures. In this paper, an evaluating method for the scanning strategy in nanosecond laser milling of axisymmetric 3D structure is proposed. Seven scanning strategies for micro cones with size range of $600-900 \mu \mathrm{m}$ are designed and the manufacturing experiments are carried out. Cone angle and symmetric error of micro cone are mainly concerned. The following conclusions were obtained.

1 . The symmetry of the micro cone is positively correlated with the symmetry coefficient SYM of the scanning strategy;

2. The scanning strategy has a certain influence on the average cone angle of micro cones, but the influence amplitude is small. The formation of the cones still conforms to the law of material removal.

The symmetry coefficient $S Y M$ proposed in this paper can be used to quantitatively evaluate the symmetry degree of scanning strategies and to predict the symmetry of the manufactured micro circular cones. The method can be extended to all the axisymmetric 3D structures. However, the diameter errors of micro cones are very large and the proposed method cannot be applied to asymmetric 3D structures. In near future, the study on improving the diameter error of micro cones and improving the shape accuracy of asymmetric 3D structures will be carried out, specifically, the mechanisms of thermal effect to shape accuracies and the secondary finishing process and post-treatment process for micro 3D structures.

\section{Acknowledgments}

This work was supported by National Natural Science Foundation of China (No. 51575191), the Fundamental Research Funds for the Central Universities (No.2017B0071, No. 2018PY12) and 2019 Guangzhou technology project (No. 201904010368).

\section{References}

[1] Mishra. S, and V. Yadava: Opt. Laser. Eng., 73, (2015) 89.

[2] Pham. D.T. et al: Proc. Inst. Mech. Eng., Part B., 218 (2004) 1 .

[3] Žemaitis. A, et al: Opt. Laser. Eng., 114, (2019) 83.

[4] Zheng. Z, et al: Opt. Commun., 424, (2018) 190.

[5] Knowles. M.R.H, et al: Int. J. Adv. Manuf. Tech., 33, (2007) 95.

[6] Chang. W, et al: Proc. SPIE, 4760, (2002) 1014.

[7] Xing. Y, et al: Ceram. Int., 44, (2018) 23035.

[8] Liess. H. and U. Reiländer: INTERNATIONAL DESIGN CONFERENCE - DESIGN 2004, Dubrovnik, (2004) p. 1203 .

[9] Xing. Y, et al: Opt. Laser. Tech., 108, (2018) 333.

[10] Lei. C, et al: Opt. Laser. Tech., 106, (2018) 47.

[11]Feng. W, et al: Opt. Laser. Tech., 111, (2019) 653.

[12] Tangwarodomnukun. V. and T. Wuttisarn: Int. J. Adv. Manuf. Tech., 92, (2017) 293.

[13] Sivarao et al: Int. J. Eng. Tech., 10, (2010) 1.

[14] Mandal.K.K, A.S. Kuar, and S. Mitra: Opt. Laser. Tech., 107, (2018) 260.

[15] Ahmed. N, et al: Materials(Basel)., 12, (2019) 1674.

[16] Janssen. S and M.C. Eckstein: J. Laser Micro/Nanoeng., 13, (2018) 36.

[17]Zhang. Y, et al: AIP. Adv., 7, (2017) 075010.

[18] Marla. D, U.V. Bhandarkar, and S.S. Joshi: Appl. Phys. A., 116, (2014) 273.

[19] Gragossian. A, et al: J. Appl. Phys., 105, (2009) 103304.

[20] Dowden. J.M: "The mathematics of thermal modeling. An introduction to the theory of laser material processing", (Chapman \& Hall/CRC,USA, 2001) p.145

(Received: October 11, 2019, Accepted: February 23, 2020) 